\title{
Analytics of Period Doubling
}

\author{
Paul E. Phillipson \\ Department of Physics, Box 390, University of Colorado, Boulder, CO 80309-0390, USA
}

\begin{abstract}
Approximate analytic solutions for periodic orbits of the quadratic map $x \rightarrow r x(1-x)$ are developed using algebraic methods. These solutions form the basis of an exact algorithm which predicts the quantitative order of periodic points characteristic of the Feigenbaum scenario. The algorithm holds for any one dimensional unimodal map. A general procedure is developed which permits calculation of period doubling parameters for large period orbits from those of low period to any desired degree of accuracy. Explicit equations are given through second order.
\end{abstract}

\section{Introduction}

The emergence of periodic points due to subharmonic bifurcations displayed by the prototypic quadratic map [1-3],

$$
x \rightarrow F_{0}(x) \equiv \operatorname{rx}(1-x),
$$

can be traced analytically using algebraic methods. The procedure to be developed is implied by the approximate renormalization method of Helleman [4-6] who was concerned with scaling. The present study is determination of periodic point structure implied by scaling. These points arise as follows: as $r$ progresses from $r_{2}=3$ a period 2 orbit which had evolved from period 1 will subsequently evolve at $r_{4}=3.44 \ldots$ to a period 4 harmonic of $F_{0}$. The period 4 harmonic are four periodic points of period 2 for $F_{0}^{2}$. At $r_{8}=3.54 \ldots$ the period 4 harmonic evolves to the period 8 harmonic of $F_{0}$ which are points of period 2 for $F_{0}^{4}$. This process of period doubling continues as $r$ increases until the accumulation point $r_{\infty}=3.5699 \ldots$ at which appears the harmonic of infinite period. This is a particular example of the Feigenbaum period doubling scenario displayed quite generally by onedimensional unimodal maps. The harmonic of period $p$, or $p$-cycle, is the orbit of $p$ 
periodic points $a_{n}^{(p)}$ given for the quadratic map by

$$
\begin{aligned}
a_{n+1}^{(p)}(r) & =r a_{n}^{(p)}(r)\left[\left(1-a_{n}^{(p)}(r)\right], \quad n=1,2, \ldots, p,\right. \\
a_{n}^{(p)}(r) & =F_{0}^{(p)}\left[a_{n}^{(p)}(r)\right], \\
a_{n+p}^{(p)}(r) & =a_{n}^{(p)}(r), \quad r_{p}<r<r_{2 p} .
\end{aligned}
$$

Equation (2a) shows how the points of the $p$-cycle arise by iteration of $F_{0},(2 \mathrm{~b})$ shows these points are fixed points of the $p$-th iterate of $F_{0}$, and (2c) shows the points define a closed $p$-cycle orbit within a specific parameter range. This range is bounded at the lower end by $r_{p}$, which is that value at which the $p / 2$-cycle becomes unstable and the $p$-cycle is born, and at the upper end by $r_{2 p}$, which is that value of $r$ at which the $p$-cycle becomes unstable and the $2 p$-cycle is born.

The first aim of the present work is development of explicit approximate analytic representation of $a_{n}^{(p)}(r)$ of (2) as functions of $r$. While this goal appears restricted insofar as it is limited to the quadratic map, the algebra of the period doubling scenario will be shown to contain predictions concerning $p$-cycles applicable in general to one dimensional unimodal maps. The reason for this is that particular as the quadratic map is and approximate as the algebraic formalism is even within the quadratic map framework, this algebra exhibits dynamical progression of period doubling bifurcations and concomitant $p$-cycle orbits characteristic of these maps. As a consequence a general algorithm for unimodal maps is developed which predicts the quantitative order of points of any $p$-cycle sequence which has evolved by period doublings from period 2 . The symbolic dynamics [7-9] for the harmonics of period 2 become, therefore, related to the structure of the periodic points themselves. The algebraic analysis also leads to an equally general method whereby period doubling parameters for large period can be calculated from those of smaller period to any desired degree of accuracy. The results, which are given through second order but extendable to higher order, might be expected to be useful in an interactive way with experimental measurements of period doubling sequences.

\section{Periodic Orbits of the Quadratic Map}

Solutions to (2) will be generated by iterates of the mapping

$$
x \rightarrow H(x) \equiv f x-\mu x^{2},
$$

where $f$ and $\mu$ are parameters such that $H=F_{0}$ when $f=\mu=r$. When the coordinate is scaled according to $x=\frac{f}{\mu} z$, (3) becomes $z \rightarrow f z(1-z)$, which has fixed points at 0 and $1-1 / f$ and periodic points of period $2\left(z_{+}, z_{-}\right)$given by

$$
z_{\varepsilon}=\frac{(f+1)+[(f+1)(f-3)]^{1 / 2} \varepsilon}{2 f}, \quad \varepsilon \pm 1,
$$

as can be verified by substitution. To these correspond, for the initial map $H$, the periodic orbit of period 2 (or 2-cycle),

$$
x_{\varepsilon}=\frac{(f+1)+[(f+1)(f-3)]^{1 / 2} \varepsilon}{2 \mu} .
$$


Setting $f=\mu=r$, the 2-cycle periodic points of the quadratic map $F_{0}$ of (2) are then given by

$$
\begin{aligned}
& a_{n}^{(2)}=\frac{(r+1)+[(r+1)(r-3)]^{1 / 2} \varepsilon_{n}^{(2)}}{2 r}, \\
& \varepsilon_{n}^{(2)}=\cos (n-1) \pi, \quad n=1,2 .
\end{aligned}
$$

For the 4-cycle of the quadratic map we return to the $H$-mapping. Since $x_{\varepsilon}$ are fixed points of $H^{2}\left(x_{\varepsilon}=H^{2}\left(x_{\varepsilon}\right)\right)$ they serve to generate periodic points of period 2 for $H^{2}$, which equivalently are periodic points of period 4 for $H$. Up to a scale factor of the type above these are precisely the points of the 4-cycle of the quadratic map. Set $x=x_{\varepsilon}+y$. Iteration of (3) results in the following mapping through terms in $y^{2}$,

$$
y \rightarrow H^{2}(y) \simeq \hat{f} y-\hat{\mu} y^{2},
$$

with

$$
\begin{gathered}
\hat{f}=-f^{2}+2 f+4, \\
\hat{\mu}=\mu\left\{(f+1)(f-3)+3[(f+1)(f-3)]^{1 / 2} \varepsilon\right\} .
\end{gathered}
$$

$\hat{f}$ is single valued in that it depends only upon its predecessor $f$, while $\mu$ is double valued in that it also depends upon $\varepsilon( \pm 1)$. The basic algebraic approximation is neglect of cubic and quartic terms in $y$ of $H^{2}$. Equation (7) has the same structure as (3), so that the same procedure which led to (5) results in the 2-cycle for $H^{2}$,

$$
y_{\hat{\varepsilon}}=\frac{(\hat{f}+1)+[(\hat{f}+1)(\hat{f}-3)]^{1 / 2} \hat{\varepsilon}}{2 \hat{\mu}}, \quad \hat{\varepsilon}= \pm 1 .
$$

Adding this result to (5) gives, for the initial map $H$, the periodic orbit of period 4 (or 4-cycle)

$$
x_{\varepsilon, \hat{\varepsilon}}=x_{\varepsilon}+y_{\hat{\varepsilon}},
$$

whose four points correspond to $x_{1,1}, x_{1,-1}, x_{-1,1}, x_{-1,-1}$. Again setting $f=\mu=r$, the 4-cycle periodic points of the quadratic map $F_{0}$ of (2) are then given by

$$
\begin{aligned}
a_{n}^{(4)}= & \frac{(r+1)+[(r+1)(r-3)]^{1 / 2} \varepsilon_{n}^{(2)}}{2 r} \\
& +\frac{\left(f_{2}+1\right)+\left[\left(f_{2}+1\right)\left(f_{2}-3\right)\right]^{1 / 2} \varepsilon_{n}^{(4)}}{2 \mu_{n}^{(2)}}, \quad[n=1,2,3,4],
\end{aligned}
$$

where $f_{2}=-r^{2}+2 r+4$ from (8), $\mu_{n}^{(2)}=r\left\{(r+1)+3[(r+1)(r-3)]^{1 / 2} \varepsilon_{n}^{(2)}\right\}$ from (9) and

$$
\begin{aligned}
& \varepsilon_{n}^{(2)}=\cos (n-1) \pi, \quad n=1,2,3,4, \\
& \varepsilon_{n}^{(4)}=\begin{array}{lll}
+1 & \text { for } & n=1,2 \\
-1 & \text { for } & n=3,4
\end{array}
\end{aligned}
$$

This procedure can be repeated to generate the 8 -cycle by setting $y=\hat{y}+y^{\prime}$ in (7), noting that $\hat{y}=H^{4}(\hat{y})$, retaining terms only through $y^{\prime 2}$ and carrying out the same 
procedure that led to $(10)$. If the process is continued the results are the periodic points of the $p$-cycle of the quadratic map (2), given in the present approximation by

$$
a_{n}^{(p)}=\sum_{\nu=1}^{k} \frac{\left(f_{m}+1\right)+\left[\left(f_{m}+1\right)\left(f_{m}-3\right)\right]^{1 / 2} \varepsilon_{n}^{(2 m)}}{2 \mu_{n}^{(m)}}, \quad\left[m=2^{v-1}, p=2^{k}\right],
$$

where $f_{m}$ and $\mu_{n}^{(m)}$ are recursively defined polynomials in $r$,

$$
\begin{gathered}
f_{2 m}=-f_{m}^{2}+2 f_{m}+4 \quad\left(f_{1}=r\right), \\
\mu_{n}^{(2 m)}=\mu_{n}^{(m)}\left\{\left(f_{m}+1\right)\left(f_{m}-3\right)+3\left[\left(f_{m}+1\right)\left(f_{m}-3\right)\right]^{1 / 2} \varepsilon_{n}^{(2 m)}\right\} \quad\left(\mu_{n}^{(1)}=r\right),
\end{gathered}
$$

with signatures $\varepsilon_{n}^{(2 m)}$ which assume the values of +1 or -1 according to (26) below. Equations (12) and (13) illustrate this result for the case of $p=4$.

Equations (14)-(16) are the desired approximate analytic solutions to (2) subject to specification of the parameter range for a given $p$-cycle. This range is characterized by windows [1] whose width is $r_{p}<r<r_{2 p}$ of (2c) such that the $p$ cycle is stable within this parameter range. Formally period doubling from period $p / 2$ to period $p$ is reflected by the last $p=2^{k}$ term in the sum of (14),

$$
a_{n}^{(p)}=a_{n}^{(p / 2)}+\frac{\left(f_{p / 2}+1\right)+\left[\left(f_{p / 2}+1\right)\left(f_{p / 2}-3\right)\right]^{1 / 2} \varepsilon_{n}^{(p)}}{2 \mu_{n}^{(p / 2)}} .
$$

This last term is zero for $0<r<r_{p}$, and begins to emerge at $r=r_{p}$. The equality is fulfilled by $f_{p / 2}\left(r_{p}\right)=-1$. Setting $m=p / 2$ in (15) gives the related result $f_{p}\left(r_{p}\right)=+1$. Within the window there is a particular $r=r_{p}^{*}$ at which the $p$-cycle is superstable [3] in that the linear term of (7) must vanish. This condition is fulfilled by $f_{p}\left(r_{p}^{*}\right)=0$. The polynomials of $(15)$ have, then, the following properties characterizing the window for period $p$ in exact parallel to the conditions on the slope of iterates of one-dimensional maps $[1,2]$

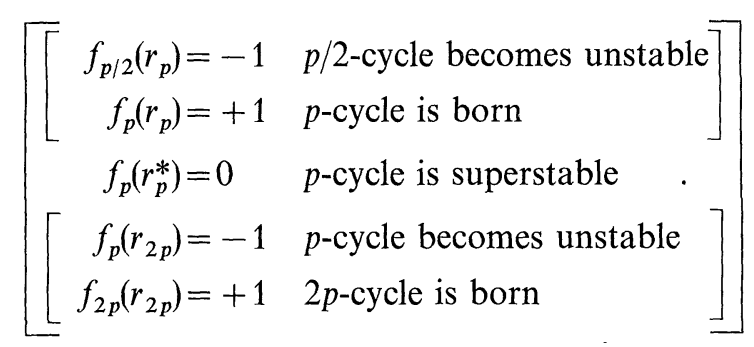

The condition $f_{p}\left(r_{p}\right)=f_{2 p}\left(r_{2 p}\right)=1$ linking the births of two adjacent cycles is a special case of the following translational symmetry principle: there always exists a pair of $r$-values $s_{p}$ and $s_{2 p}$ such that $f_{p}\left(s_{p}\right)$ in the $p$-window is reproduced by $f_{2 p}\left(s_{2 p}\right)$ in the $2 p$-window,

$$
f_{p}\left(s_{p}\right)=f_{2 p}\left(s_{2 p}\right)=\lambda \quad(\text { say }), \quad p=2,4, \ldots
$$

This provides the basis for analytic determination of the period doubling parameters $r_{p}$ and the superstable parameters $r_{p}^{*}$ which quantitatively bound and characterize the $p$-cycle windows. From (15) setting $\lambda=f_{2}\left(s_{2}\right)$ with $f_{1}=s_{2}$ yields 
$s_{2}=1+(5-\lambda)^{1 / 2}$. Successively setting $\lambda=f_{2 p}\left(s_{2 p}\right), \lambda=f_{p}\left(s_{2 p}\right) \ldots$ gives

$$
\begin{gathered}
f_{2 p}\left(s_{2 p}\right)=2-s_{2}, \quad f_{p / 2}\left(s_{2 p}\right)=1-\left(3+s_{2}\right)^{1 / 2} ; \\
f_{p / 4}\left(s_{2 p}\right)=1-\left(s_{4}+3\right)^{1 / 2}, \quad s_{4}=1+\left(3+s_{2}\right)^{1 / 2} ; \\
f_{p / 8}\left(s_{2 p}\right)=1-\left(s_{8}+3\right)^{1 / 2}, \quad s_{8}=1+\left(3+s_{4}\right)^{1 / 2} ; \\
\cdots \\
f_{2}\left(s_{2 p}\right)=-s_{2 p}^{2}+2 s_{2 p}+4=2-s_{p} .
\end{gathered}
$$

Solution of the quadratic equation ending this progression gives a simple equation which links parameter values of adjacent windows in a continuous way as a function of $\lambda$,

$$
\begin{aligned}
s_{2 p}(\lambda) & =1+\left[3+s_{p}(\lambda)\right]^{1 / 2}, \quad p=2,4, \ldots, \\
s_{2}(\lambda) & =1+(5-\lambda)^{1 / 2} .
\end{aligned}
$$

Generation of $r_{p}$ and $r_{p}^{*}$ correspond to the particular values of $\lambda=1$ and 0 respectively, so that

$$
\begin{array}{ll}
r_{2 p}=1+\left(3+r_{p}\right)^{1 / 2} & \left(s_{1}=r_{1}=1\right), \\
r_{2 p}^{*}=1+\left(3+r_{p}^{*}\right)^{1 / 2} & \left(s_{1}=r_{1}^{*}=2\right) .
\end{array}
$$

The values from this result of $r_{2}=3, r_{2}^{*}=1+\sqrt{5}=3.23606 \ldots, r_{4}=1+\sqrt{6}$ $=3.44948 \ldots$ are exact, but this generating relation is a consequence of confining the scaled maps of successive period doublings to quadratic form. Necessarily the higher $p$-cycle parameters are approximate. Table 1 shows a comparison of (21) with computer results. Agreement is within one unit in the second significant figure. As $p \rightarrow \infty$ the accumulation point is reached for which $s_{2 p}=s_{p}=r_{\infty}$ (independent of $\lambda$ ) which solution of $(20)$ predicts to be $r_{\infty}=(3+\sqrt{17}) / 2=3.5615 \ldots$ previously noted [5], compared with the computer value of $3.56994 \ldots$ [3].

The above scheme predicts the width of a $p$-cycle window of the quadratic map which has evolved by period doubling from period 2 is given approximately by

$$
\begin{gathered}
r_{p}<r<1+\left(3+r_{p}\right)^{1 / 2}, \\
r_{1}=1, \quad r_{\infty}=\frac{3+\sqrt{17}}{2}=3.5615 \ldots .
\end{gathered}
$$

The concomitant evolution of the periodic points according to (14) reflects progression of $f$-polynomials recursively defined by (15). Each polynomial is subject to the step-like condition $f_{p}(r)=0$ if $0<r<r_{2 p}$, but $f_{p}\left(r_{2 p}\right)=-1$. Between $r_{2 p}$ and $r_{\infty} f_{p}$ is a smoothly decreasing function of $r$. As $r \rightarrow r_{\infty}$ all polynomials (except $f_{1}=r_{\infty}$ ) converge to the same value $f_{\infty}$ found by formally setting $f_{2 m}=f_{m}=f_{\infty}$ in (15) and solving the resultant quadratic equation. This gives $f_{\infty}=f_{p}\left(r_{\infty}\right)=(1-\sqrt{17}) / 2=-1.5615 \ldots$ for all $p$. As a consequence all polynomials in their non-zero range are bounded by $-1 \leqq f_{p}(r) \leqq-1.5615 \ldots, r_{2 p} \leqq r \leqq r_{\infty}$. Table 2 shows a representative calculation of the 16-supercycle according to (14), 
Table 1. Period doubling parameters $\left(r_{p}\right)$ and superstable parameters $\left(r_{p}^{*}\right)$ for the quadratic map $x \rightarrow r x(1-x)$

\begin{tabular}{llllll}
\hline Period $p$ & \multicolumn{2}{l}{ Computer $^{\mathrm{a}}$} & \multicolumn{3}{l}{ Theory $^{\mathrm{b}}$} \\
\cline { 2 - 3 } & $r_{p}^{*}$ & $r_{p}$ & & $r_{p}^{*}$ & $r_{p}$ \\
\cline { 5 - 6 } 1 & 2 & & & 2 & 3 \\
2 & 3.2361 & 3 & & 3.2361 & 3.4495 \\
4 & 3.4986 & 3.4495 & & 3.4972 & 3.5396 \\
8 & 3.5546 & 3.5441 & & 3.5490 & 3.5573 \\
16 & 3.5667 & 3.5644 & & 3.5591 & \\
32 & 3.5692 & 3.5688 & & 3.5611 & 3.5607 \\
64 & 3.5699 & 3.5697 & & 3.5616 & 3.5614 \\
& & 3.5699 & & 3.5616 \\
\hline
\end{tabular}

\footnotetext{
${ }^{\mathrm{a}} r_{p}$ and $r_{p}^{*}$ are those values of $r$ for which the slope of the $p$-th iterate of $(1)$ is -1 and 0 , respectively. The supercycle at $r_{p}^{*}$ always contains $1 / 2$ as one of the cycle elements (cf. [3]). Computer data for $r_{p}$ from Collet and Eckmann [8, Table I.25]. $\mu_{n}$ of this reference is related to the present $r_{p}$ by $r_{p}=1+\left(1+4 \mu_{n}\right)^{1 / 2}$, $p=2^{n+1}$

${ }^{\mathrm{b}}$ Equations $(21 \mathrm{a}, \mathrm{b}): r_{2}^{*}=1+\sqrt{5}=3.23606 \ldots$ and $r_{4}=1+\sqrt{6}$ $=3.44948 \ldots$ are exact
}

compared to computer solution of (1). In every $p$-cycle the supercycle at $r_{p}^{*}$, at which the slope of the map is zero, always contains $a^{*}=1 / 2$ (for the quadratic map) as a cycle element $[3,7]$. For the ordering (26) this corresponds to $a_{p}^{(p)}\left(r_{p}^{*}\right)$, or the last entry in Table 2. The departure of the theoretical value of 0.5093 from 0.5 finds its roots in the approximate value of $r_{16}^{*}$ computed from (21b) and from the fact that $a^{*}$ arises from a multiplicative chain rule approximated here by sums arising from successive renormalizations. Quantitatively theory and computer disagree by no more than two units in the second significant figure. Two generic measures of quantitative error are: (1) the Feigenbaum ratio $\delta$ which is the ratio between adjacent $r_{p}$ 's as $p \rightarrow \infty$, and (2) the scaling constant $\alpha$ which is the ratio of the difference between elements $a_{p}^{* *}$ lying closest to $a^{*}$, and $a^{*}$, for successive periods as $p \rightarrow \infty$ [3]. The ordering (26) implies $a_{p}^{* *}=a_{p / 2}^{(p)}\left(r_{p}^{*}\right)$. Noting that numerically $f_{\infty}+r_{\infty}=2$, one finds from (14) and (20) the result

$$
\begin{aligned}
\delta & =\operatorname{Lim}_{p \rightarrow \infty} \frac{\left(r_{2 p}-r_{p}\right)}{\left(r_{4 p}-r_{2 p}\right)}=\left.\frac{d s_{2 p}}{d s_{p}}\right|_{r_{\infty}}=1+\sqrt{17}=5.1231 \ldots(4.66920 \ldots), \\
\alpha & =\operatorname{Lim}_{p \rightarrow \infty}-\left[\frac{\left(a_{p}^{* *}-a^{*}\right)}{\left(a_{2 p}^{* *}-a^{*}\right)}\right]=-\left.\left\{(r+1)(r-3)-3[(r+1)(r-3)]^{1 / 2}\right\}\right|_{r_{\infty}} \\
& =2.2399 \ldots(2.50290 \ldots),
\end{aligned}
$$


Table 2. Period 16 supercycle

\begin{tabular}{|c|c|c|c|c|c|c|}
\hline \multicolumn{3}{|c|}{ Periodic points $a_{n}^{(16)}$} & \multicolumn{4}{|c|}{ Signatures $^{c}$} \\
\hline$n$ & Computer $^{a}$ & Theory $^{b}$ & $\varepsilon_{n}^{(2)}$ & $\varepsilon_{n}^{(4)}$ & $\varepsilon_{n}^{(8)}$ & $\varepsilon_{n}^{(16)}$ \\
\hline 1 & 0.8917 & 0.8875 & 1 & 1 & 1 & 1 \\
\hline 2 & 0.3445 & 0.3419 & -1 & 1 & 1 & 1 \\
\hline 3 & 0.8055 & 0.8145 & 1 & -1 & 1 & 1 \\
\hline 4 & 0.5589 & 0.5807 & -1 & -1 & 1 & 1 \\
\hline 5 & 0.8793 & 0.8784 & 1 & 1 & -1 & 1 \\
\hline 6 & 0.3785 & 0.3717 & -1 & 1 & -1 & 1 \\
\hline 7 & 0.8390 & 0.8439 & 1 & -1 & -1 & 1 \\
\hline 8 & 0.4817 & 0.4845 & -1 & -1 & -1 & 1 \\
\hline 9 & 0.8905 & 0.8867 & 1 & 1 & 1 & -1 \\
\hline 10 & 0.3479 & 0.3445 & -1 & 1 & 1 & -1 \\
\hline 11 & 0.8091 & 0.8171 & 1 & -1 & 1 & -1 \\
\hline 12 & 0.5508 & 0.5724 & -1 & -1 & 1 & -1 \\
\hline 13 & 0.8824 & 0.8807 & 1 & 1 & -1 & -1 \\
\hline 14 & 0.3700 & 0.3640 & -1 & 1 & -1 & -1 \\
\hline 15 & 0.8314 & 0.8363 & 1 & -1 & -1 & -1 \\
\hline 16 & 0.5000 & 0.5093 & -1 & -1 & -1 & -1 \\
\hline
\end{tabular}

${ }^{a}$ Equation (1) with $r=3.56667$

${ }^{\mathrm{b}}$ Equation (14) with $r_{16}^{*}=3.55909$ from (21 b)

${ }^{\mathrm{c}}$ Equation (26)

where the numbers in parentheses are Feigenbaum's numerical results. The analytic values, also reported by Helleman $[4,5]$, are about $10 \%$ in discrepancy with the computer values, these quantities reflecting as they do the ratio of small differences of relatively large numbers.

\section{Symbolic Dynamics of Period Doubling}

Period doubling is characterized by the splitting of each cycle element into two components at specific parameter values $r_{p}$, where $r$ parametrizes the map. Equation (17) is an expression of this behaviour for the quadratic map. The splitting is formally reflected by the signatures: period doubling from $p / 2$ means that $\varepsilon_{n}^{(p / 2)}$ in the $p$-cycle periodic points sequence repeats itself twice while $\varepsilon_{n}^{(p)}$ runs through its sequence once. Subsequent splitting to form the $2 p$-cycle means that $\varepsilon_{n}^{(p / 2)}$ now runs through its sequence four times, $\varepsilon_{n}^{(p)}$ runs through its sequence twice and the new signatures $\varepsilon_{n}^{(2 p)}$ run through the sequence once, and so on. An example of this is $(13)$ for period 4 : the $\varepsilon_{n}^{(2)}$ sequence is $(1,-1,1,-1)$ or $(1,-1)$ twice and the $\varepsilon_{n}^{(4)}$ sequence is $(1,1,-1,-1)$ once. The signatures of the 16 -(super) cycle shown in Table 2 further illustrate this trend: here the $\varepsilon_{n}^{(2)}$ sequence is repeated eight times, the $\varepsilon_{n}^{(4)}$ sequence four times, the $\varepsilon_{n}^{(8)}$ sequence two times and the $\varepsilon_{n}^{(16)}$ sequence once. From this point of view period doubling represents the unfolding of signatures $\varepsilon_{n}^{(2 m)}= \pm 1(m=1,2,4, \ldots, p / 2 ; n=1,2,4, \ldots, p)$. This suggests an analysis of symbolic dynamics of period doubling in terms of a signature construction whose 
sequences of allowable values \pm 1 are subject to (1) rules which allow prediction of the ordering of the $p$-cycle elements as they arise through iteration of the map, and (2) rules which allow prediction of the relative magnitudes of the cycle elements. To be more precise, the question that will be considered is the following: presuming a p-cycle has evolved from a 2 -cycle by the period doubling route what is the relationship between the location of a cycle element in the iterative ordering generated by the map and its magnitude relative to those of the other cycle elements?

Consider a unimodal map $x \rightarrow F(r, x)$ parametrized by $r$ whose periodic points arise by iteration (I-order) according to

$$
\begin{array}{ll}
a_{n+1}^{(p)}=F\left(r, a_{n}^{(p)}\right), & n=1,2, \ldots, p, \\
a_{n+p}^{(p)}=a_{n}^{(p)}, & p=2,4, \ldots \quad(I \text {-order of the } p \text {-cycle }) .
\end{array}
$$

Equation (2) is the special case of the $I$-order for the quadratic map with $F=F_{0}$. The cycle elements can also be quantitatively arranged in a series numerically largest to numerically smallest ( $Q$-order) according to

$$
\begin{aligned}
& a_{n}^{\prime(p)}>a_{n+1}^{\prime(p)}, \\
& a_{1}^{(p)}>a_{2}^{\prime(p)}>\ldots>a_{p}^{(p)} \quad(Q \text {-order of the } p \text {-cycle }),
\end{aligned}
$$

so the above question can be restated: given the $I$-order can one predict the $Q$ order and vice-versa? Consider iteration according to (24) of the largest element of the $p$-cycle, which by convention will be fixed at $n=1$, so that $a_{\max }^{(p)} \equiv a_{1}^{\prime(p)}=a_{1}^{(p)}$. A general feature of unimodal maps is that the iterate of the largest element is always the smallest element, so that $a_{\mathrm{min}}^{(p)}=a_{p}^{\prime}=a_{2}^{(p)}$. This result is a consequence of $F$ having an assumed normal quadratic maximum. A corollary of this is that if $a^{*}$ is the common cycle element for all $p$-cycles at their appropriate superstable parameter values (e.g. $a^{*}=\frac{1}{2}$ at $r_{p}^{*}$ for the quadratic map), then iteration of $a^{*}$ is always $a_{\max }^{(p)}=a_{1}^{(p)}$. This implies $a^{*}$ is the last element $a_{p}^{(p)}$ when $r=r_{p}^{*}$. The iterative ordering $\left(a^{*}, a_{\max }^{(p)}, a_{\min }^{(p)}\right)$ is implied by the inverse path discussion of Metropolis, Stein, and Stein [7]. It holds also for basic periods greater than two and their harmonics, such as $r^{*}$ values beyond the accumulation point for the quadratic map $\left(r_{\infty}<r \leqq 4\right)$. Another example is provided by the period six supercycles of the sine map shown in Table 3. Regardless of the order of the other elements this triplet

Table 3. Period six supercycles $a_{n}^{(6)}$ of the sine map: $x \rightarrow r \sin \pi x^{a}$

\begin{tabular}{llllll}
\hline$r^{*}$ & 0.8811406 & 0.9435875 & 0.9735656 & 0.9892022 & 0.9982647 \\
\hline$n$ & & & & & \\
$6\left(a^{*}\right)$ & 0.5000 & 0.5000 & 0.5000 & 0.5000 & 0.5000 \\
$1(\max )$ & 0.8811 & 0.9436 & 0.9736 & 0.9892 & 0.9983 \\
$2(\min )$ & 0.3214 & 0.1664 & 0.0808 & 0.0335 & 0.0054 \\
3 & 0.7461 & 0.4710 & 0.2444 & 0.1041 & 0.0171 \\
4 & 0.6307 & 0.9397 & 0.6761 & 0.3177 & 0.0535 \\
5 & 0.8079 & 0.1778 & 0.8283 & 0.8313 & 0.1670 \\
\hline
\end{tabular}

${ }^{a} r^{*}$ values from Metropolis et al. [7] 
sequence is always observed. The $I$-order of all the elements of a $p$-cycle will be fixed by formally introducing signatures $\varepsilon_{n}^{(2 m)}$ such that any element of the $p$-cycle is uniquely specified by the values $\left(\varepsilon_{n}^{(2)}, \varepsilon_{n}^{(4)} \ldots \varepsilon_{n}^{(p)}\right)$ assigned by the following rule:

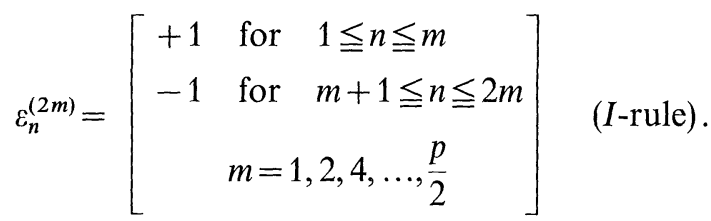

The $I$-order of the $p$-cycle elements $a_{n}^{(p)}$ is constructed by an $I$-table, exemplified by Table 4 for period 8 , for which the rule for each $\varepsilon_{n}^{(2 m)}$ is repeated $p / 2 m$ times. This is the repeating sequence discussed above, and in particular is the sequence of signatures for the 16-cycle of Table 2 . While the signature sequence entered into the approximate analytic expression for the periodic points, it now symbolically orders the periodic points by iteration for any harmonic of period 2 for any unimodal map. It is to be noted that $a_{\max }^{(p)}$ corresponds to all signatures $+1, a^{*}$ at $r_{p}^{*}$ corresponds to all signatures equal to -1 and $a_{\min }^{(p)}$ corresponds to $\varepsilon_{n}^{(2)}=-1$ and all remaining signatures equal to +1 . The $Q$-order of the $p$-cycle elements is similarly determined by the signatures $\varepsilon_{n}^{(2 m)}$ such that any element is uniquely specified by values $\left(\varepsilon_{n}^{(2)}, \varepsilon_{n}^{(4)} \ldots \varepsilon_{n}^{(p)}\right)$ assigned now by the rule

$$
\left.\varepsilon_{n}^{(2 m)}=\left[\begin{array}{ccc}
+1 & \text { for } & 1 \leqq n \leqq \frac{p}{2 m} \\
-1 & \text { for } & \frac{p}{2 m}+1 \leqq n \leqq \frac{3 p}{2 m} \\
+1 & \text { for } & \frac{3 p}{2 m}+1 \leqq n \leqq \frac{5 p}{2 m} \\
-1 & \text { for } & \frac{5 p}{2 m}+1 \leqq n \leqq \frac{7 p}{2 m} \\
& & \vdots
\end{array}\right] \text { (Q-rule }\right)
$$

where this sequence ends at that value of $k$ for which $(2 k+1) p / 2 m \geqq p$, or $(2 k+1) \geqq 2 m$. For each $m$ there must be a total of $p$ signatures, and after the sequence terminates at $(2 k-1) p / 2 m$, the remaining $p(2 m+1-2 k) / 2 m$ entries are of opposite sign to that of this last member of the sequence. The $Q$-order of the $p$-cycle elements $a_{n}^{\prime(p)}$ is constructed by a $Q$-table as shown in Table 4 for period 8 . By comparison of the $I$-table with the $Q$-table one can identify the $a$-element of the $I$ order with that element of the $Q$-order which has the same signature sequence $\left(\varepsilon_{n}^{(2)}, \varepsilon_{n}^{(4)} \ldots \varepsilon_{n}^{(p)}\right)$. The result is a correlation table, shown for period 8 in Table 4 . The identification of a given $a_{n}^{(p)}$ with the appropriate $a_{n^{\prime}}^{(p)}$ predicts that the $n$-th iterate of the $p$-vycle with respect to $a_{1}^{(p)}=a_{\max }^{(p)}$ has the $n^{\prime}$-th largest value of the $p$ possible values of the cycle elements. The numbers shown in the last column of Table 4 , computed from within the period 8 window of the sine map, is included to provide a concrete example of this result. Other conclusions can be drawn concerning $p$ cycle fixed points which arise from successive doublings originating from period 
Table 4. $I, Q$, and correlation tables for period 8

\begin{tabular}{|c|c|c|c|c|c|c|c|c|}
\hline \multicolumn{4}{|c|}{$I$-table ${ }^{\mathrm{a}}$} & \multicolumn{4}{|c|}{$Q$-table ${ }^{\mathrm{b}}$} & \multirow[t]{2}{*}{ Correlation table ${ }^{\mathrm{c}}$} \\
\hline$a_{n}^{(8)}$ & $\varepsilon_{n}^{(2)}$ & $\varepsilon_{n}^{(4)}$ & $\varepsilon_{n}^{(8)}$ & $a_{n}^{\prime(8)}$ & $\varepsilon_{n}^{(2)}$ & $\varepsilon_{n}^{(4)}$ & $\varepsilon_{n}^{(8)}$ & \\
\hline$a_{1}$ & 1 & 1 & 1 & $a_{1}^{\prime}$ & 1 & 1 & 1 & $a_{1}=a_{1}^{\prime}(0.860)-\max$ \\
\hline$a_{2}$ & -1 & 1 & 1 & $a_{2}^{\prime}$ & 1 & 1 & -1 & $a_{2}=a_{8}^{\prime}(0.367)-\min$ \\
\hline$a_{3}$ & 1 & -1 & 1 & $a_{3}^{\prime}$ & 1 & -1 & -1 & $a_{3}=a_{4}^{\prime}(0.786)$ \\
\hline$a_{4}$ & -1 & -1 & 1 & $a_{4}^{\prime}$ & 1 & -1 & 1 & $a_{4}=a_{5}^{\prime}(0.536)$ \\
\hline$a_{5}$ & 1 & 1 & -1 & $a_{5}^{\prime}$ & -1 & -1 & 1 & $a_{5}=a_{2}^{\prime}(0.854)$ \\
\hline$a_{6}$ & -1 & 1 & -1 & $a_{6}^{\prime}$ & -1 & -1 & -1 & $a_{6}=a_{7}^{\prime}(0.380)$ \\
\hline$a_{7}$ & 1 & -1 & -1 & $a_{7}^{\prime}$ & -1 & 1 & -1 & $a_{7}=a_{3}^{\prime}(0.800)$ \\
\hline$a_{8}$ & -1 & -1 & -1 & $a_{8}^{\prime}$ & -1 & 1 & 1 & $a_{8}=a_{6}^{\prime}(0.507)$ \\
\hline
\end{tabular}

${ }^{a}$ Signatures $\varepsilon_{n}^{(m)}$ from $I$-rule of (26). The superscript (8) is implied in the listing of fixed points: $a_{3}=a_{n}^{(8)}, n=3$, etc.

${ }^{\mathrm{b}}$ Signatures $\varepsilon_{n}^{(m)}$ from $Q$-rule of (27). The superscript (8) is implied in the listing of fixed points: $a_{3}^{\prime}=a_{n}^{\prime(8)}, n=3$, etc.

${ }^{\mathbf{c}}$ Numerical values are to show a typical illustration. Computed from the sine map of Table 3 at $r=0.86$

two. For example, since $a_{\max }^{(p)}$ is at $n=1$ by convention then necessarily the second to largest fixed point is at $n=1+p / 2$. It is a simple matter to construct such generic tables from the rules of (26) and (27) applicable to any one dimensional unimodal map for any period of interest. Table 5 gives the correlation table for periods 4 through 32 .

The present results hold throughout a parameter range restricted to harmonics of period 2. Periodic points for basic periods greater than 2 and their harmonics can be quantitatively ordered in a similar way on the basis of signatures whose rules generalize the constructions of (26) and (27). The present rules, however, if applied at the supercycle points of p-cycles permit quantification of the $U$ sequence of Metropolis, Stein, and Stein [7] for harmonics of period 2. The MSS construction states that since for every $p$-cycle the supercycle at $r_{p}^{*}$ always contains $a^{*}$ as a cycle element, then with respect to $a^{*}$ all other elements are either greater than $(R)$ or less than $(L) a^{*}$. Each $p$-cycle sequence thus corresponds to a word of finite length made of $R$ 's and L's. Word construction rests on the rule that if a word $P$ characterizes a $p$-cycle, then $P X P$ is the word characterizing the $2 p$-cycle adjacent to $P . X=R$ if there is an even number of $R$ 's in $P$ and $x=L$ if there are an odd number of $R$ 's in $P$. The $U$-sequence with quantitative ordering can be read from the entries in Table 5. From the $I$-order table the last entry corresponds to $a^{*}=a_{p}^{(p)}$. From the $Q$-order table for a given $p$ all elements above the $a_{p}^{(p)}$ entry are $R$ elements (whose largest entry is $a_{\text {max }}$ at $n=1$ ), and all below are $(L)$ elements (whose smallest entry is $a_{\min }$ at $n=p$ ). For example, consider period 8 . From the $I$-order table $a^{*}=a_{6}^{\prime}$, where the superscript "(8)" is implied. From the $Q$-order table $\left(R_{1}, R_{2}, R_{3}, R_{4}, R_{5}\right)=\left(a_{1}, a_{5}, a_{7}, a_{3}, a_{4}\right)$ and $\left(L_{1}, L_{2}\right)=\left(a_{6}, a_{2}\right)$, where $R_{k}>R_{k+1}$ and $L_{k}>L_{k+1}$. Then the $U$-sequence can be read from the $I$-order table $\left(a^{*}=a_{8}, a_{1}, a_{2}, a_{3}, a_{4}, a_{5}, a_{6}, a_{7}\right)=\left(a^{*}, R_{1}, L_{2}, R_{4}, R_{5}, R_{2}, L_{1}, R_{3}\right)$. Similarly, the $U$ sequence for any $p$-cycle can be found from Table 5 and its extension to higher periods. 
Table 5. Correlation table for periods $4,8,16$, and 32

\begin{tabular}{|c|c|c|c|c|c|c|c|c|c|}
\hline \multicolumn{5}{|c|}{ Serial $I$-order ${ }^{\mathrm{a}}$} & \multicolumn{5}{|c|}{ Serial $Q$-order ${ }^{\mathrm{a}}$} \\
\hline$a_{n}^{(p)}$ & $a_{n}^{\prime(4)}$ & $a_{n}^{\prime(8)}$ & $a_{n}^{\prime(16)}$ & $a_{n}^{\prime(32)}$ & $a_{n}^{\prime(p)}$ & $a_{n}^{(4)}$ & $a_{n}^{(8)}$ & $a_{n}^{(16)}$ & $a_{n}^{(32)}$ \\
\hline 1 & 1 & 1 & 1 & 1 & 1 & 1 & 1 & 1 & 1 \\
\hline 2 & 4 & 8 & 16 & 32 & 2 & 3 & 5 & 9 & 17 \\
\hline 3 & 2 & 4 & 8 & 16 & 3 & 4 & 7 & 13 & 25 \\
\hline 4 & 3 & 5 & 9 & 17 & 4 & 2 & 3 & 5 & 9 \\
\hline 5 & & 2 & 4 & 8 & 5 & & 4 & 7 & 13 \\
\hline 6 & & 7 & 13 & 25 & 6 & & 8 & 15 & 29 \\
\hline 7 & & 3 & 5 & 9 & 7 & & 6 & 11 & 21 \\
\hline 8 & & 6 & 12 & 24 & 8 & & 2 & 3 & 5 \\
\hline 9 & & & 2 & 4 & 9 & & & 4 & 7 \\
\hline 10 & & & 15 & 29 & 10 & & & 12 & 23 \\
\hline 11 & & & 7 & 13 & 11 & & & 16 & 31 \\
\hline 12 & & & 10 & 20 & 12 & & & 8 & 15 \\
\hline 13 & & & 3 & 5 & 13 & & & 6 & 11 \\
\hline 14 & & & 14 & 28 & 14 & & & 14 & 27 \\
\hline 15 & & & 6 & 12 & 15 & & & 10 & 19 \\
\hline 16 & & & 11 & 21 & 16 & & & 2 & 3 \\
\hline 17 & & & & 2 & 17 & & & & 4 \\
\hline 18 & & & & 31 & 18 & & & & 20 \\
\hline 19 & & & & 15 & 19 & & & & 28 \\
\hline 20 & & & & 18 & 20 & & & & 12 \\
\hline 21 & & & & 7 & 21 & & & & 16 \\
\hline 22 & & & & 26 & 22 & & & & 32 \\
\hline 23 & & & & 10 & 23 & & & & 24 \\
\hline 24 & & & & 23 & 24 & & & & 8 \\
\hline 25 & & & & 3 & 25 & & & & 6 \\
\hline 26 & & & & 30 & 26 & & & & 22 \\
\hline 27 & & & & 14 & 27 & & & & 30 \\
\hline 28 & & & & 19 & 28 & & & & 14 \\
\hline 29 & & & & 6 & 29 & & & & 10 \\
\hline 30 & & & & 27 & 30 & & & & 26 \\
\hline 31 & & & & 11 & 31 & & & & 18 \\
\hline 32 & & & & 22 & 32 & & & & 2 \\
\hline
\end{tabular}

${ }^{\text {a }}$ Entries refer to the subscript $n$ determined according to the rules (26) and (27). For example, a reading across of the seventh entry shows the seventh element $I$-ordered according to (24) has the third, fifth, and ninth largest value within the windows of periods $p=8,16$, and 32 respectively. On the other hand, the seventh to largest element appears at $n=6,11$, and 21 within the windows of periods $p=8,16$, and 32 respectively, where $n=1$ is the largest of the allowable values within any $p$-cycle, and the successive elements arise by iteration of the map according to (24)

\section{Parameter Spectrum for Period Doubling Sequences}

Equation (20) suggests the construction of an expansion linking parameters $s_{p}$ based upon the Feigenbaum ratio. This allows prediction of the spectrum of values $s_{1}, s_{2}, \ldots, s_{p}$ for any $p$ in terms of the lower few $s_{1}, s_{2}, \ldots$. In particular, if $s_{1}, s_{2}, \ldots$ are 
known, one can obtain an estimate of the accumulation point. Assume the existence of a functional relation

$$
s_{2 p}=G\left(s_{p}\right)
$$

linking parameter values of adjacent windows. $G$ of $(20)$ is the approximate function for the quadratic map. Particular values of $s_{p}\left(s_{2 p}\right)$ are the bifurcation parameters $r_{p}\left(r_{2 p}\right)$ or the parameters locating supercycles $r_{p}^{*}\left(r_{2 p}^{*}\right)$. As $p \rightarrow \infty$ all points converge to the accumulation point $r_{\infty}$, whose value in principle is given by $r_{\infty}=G\left(r_{\infty}\right)$. Assume that $s_{p}=r_{\infty}+\gamma_{p}$. Insertion into (28) and expanding around $\gamma_{p}=0$ gives

$$
\gamma_{2 p}=a \gamma_{p}+b \gamma_{p}^{2}+O \gamma_{p}^{3}, \quad a=G^{\prime}\left(r_{\infty}\right)=\frac{1}{\delta}, \quad b=\frac{1}{2} G^{\prime \prime}\left(r_{\infty}\right)
$$

Identification of $F^{\prime}\left(r_{\infty}\right)$ with the reciprocal of the Feigenbaum ratio follows from the latter's definition [cf. (23)]. The solution for $\gamma_{p}$ through second order is given by

$$
\begin{gathered}
\gamma_{p}=\gamma_{0} a^{k}+b \gamma_{0}^{2} a^{k-1}\left[\frac{1-a^{k}}{1-a}\right]+O\left(\gamma_{0}^{3}\right), \\
{\left[p=2^{k}, a=\frac{1}{\delta}=0.214169 \ldots\right],}
\end{gathered}
$$

as can be verified by substitution. The quantities $\gamma_{0}$ and $b$ are determined in terms of $s_{1}$ and $s_{2}$ according to $s_{1}=r_{\infty}+\gamma_{0}, s_{2}=r_{\infty}+\gamma_{0} a+\gamma_{0}^{2} b$, which leads to the result

$$
s_{2^{k}}=\left\{r_{\infty}-\frac{\left[r_{\infty}-s_{1}\right]}{\delta^{k}}\right\}-\left\{\left[\delta\left(r_{\infty}-s_{2}\right)-\left(r_{\infty}-s_{1}\right)\right]\left[\frac{\delta}{\delta-1}\right]\left[\frac{\delta^{k}-1}{\delta^{2 k}}\right]\right\} .
$$

The terms of the first bracket are of first order $\left(\gamma_{0}\right)$ and those of the second bracket are of second order $\left(\gamma_{0}^{2}\right)$. They require respectively knowledge of $s_{1}$ and $s_{2}$. Solution of (29) to the $n$-th order would require specification of $s_{v}, v=2^{n-1}$ and values of $s_{p}$ for $p>v$ could be calculated from this formula. For example if $s_{1}, s_{2}$ are the first two bifurcation parameters $r_{1}, r_{2}$ of some map or the result of experiment, (31) provides an estimate of $r_{p}, p=4,8, \ldots(k=2,3, \ldots)$. As will be shown elsewhere, having found $r_{4}$ solution of (29) can be extended to include third order. In principle, carrying out this procedure to all orders would effect solution of (28) for the map or the experimental situation implied by this equation. Equation (20) represents a summation of such an infinite series approximately appropriate to the quadratic map. Equation (31) can be used in an empirical way to estimate parameters of progressively higher periods, improving the formula to higher order accuracy as these parameters become certain within acceptable quantitative error. This procedure assumes knowledge of the accumulation point, for parameters $s_{p}$ enter relative to $r_{\infty}$ as $\left(r_{\infty}-s_{p}\right)$. Suppose the accumulation point is not known. Then one reverses the situation and finds $r_{\infty}$ in terms of $s_{1}, s_{2}, \ldots, s_{v}, v=2^{n-1}$, where $n$ is the order to which solution has been effected to (28). Setting $k=2$ in (31) produces 
the following expression for the accumulation point to first and second orders:

$$
\begin{gathered}
r_{\infty}=s_{1}+\frac{\delta\left(s_{2}-s_{1}\right)}{\delta-1}=s_{1}+(1.27253 \ldots)\left(s_{2}-s_{1}\right) \quad \text { [FIRST ORDER] } \\
r_{\infty}=s_{1}+\frac{\delta\left(s_{2}-s_{1}\right)}{\delta-1}\left[1-\frac{\delta}{\delta^{2}-1}\right]+\frac{\delta^{3}\left(s_{4}-s_{2}\right)}{\left[(\delta+1)(\delta-1)^{2}\right]} \\
=s_{1}+(0.98689 \ldots)\left(s_{2}-s_{1}\right)+(1.33371 \ldots)\left(s_{4}-s_{2}\right) \\
{[\delta=4.6692016 \ldots] \quad[\text { SECOND ORDER }]}
\end{gathered}
$$

The same formulae are applicable to conservative Feigenbaum sequences with $\delta=8.7210972 \ldots$ [4]. The first order terms in (31) and the corresponding estimate of the accumulation point (32a) have been given by Collet and Eckmann [8] and Helleman [4] respectively. It is to be noted how the coefficient of $\left(s_{2}-s_{1}\right)$ goes below unity in second order with a compensating $\left(s_{4}-s_{2}\right)$ contribution. As an example, using the computer values of $s_{p}=r_{p}, p=2,4,8$ for the quadratic map results in first and second order accumulation point values of 3.5720 and 3.5698 respectively to be compared with the computer value of 3.56995 . It is often computationally simpler to determine the superstable parameters for which the estimate according to (32b) is at least as reliable. For example, using $s_{p}=r_{p}^{*}$ values for the quadratic map [7] results for the accumulation point in the poorer value in first order of 3.5729 but a superior second order value of 3.56996 compared to the above. Investigations indicate the accumulation point is satisfactorily determined in second order using (32b) from which the period doubling parameters can be estimated using (31) and its extension to third order.

\section{References}

1. May, R.M.: Simple mathematical models with very complicated dynamics. Nature 261, 459-467 (1976)

2. Feigenbaum, M.J.: Quantitative universality for a class of nonlinear transformations. J. Stat. Phys. 19, 25-52 (1978)

3. Schuster, H.G.: Deterministic chaos, an introduction, Chap. 3. Weinheim: Physik-Verlag 1984

4. Helleman, R.H.G.: Self-generated chaotic behavior in nonlinear mechanics. In: Fundamental problems in statistical mechanics, Vol. 5. Cohen, E.G.D. (ed.), pp. 165-233. Amsterdam, New York: North-Holland 1980

5. Helleman, R.H.G., MacKay, R.S.: One mechanism for the onsets of large-scale chaos in conservative and dissipative systems. In: Long-time prediction in dynamics. Horton, C.W., Jr., Reichl, L.E., Szebehely, V.G. (eds.), pp. 95-126. New York: Wiley 1983

6. Lichtenberg, A.J., Lieberman, M.A.: Regular and stochastic motion, Chap. 7. Berlin, Heidelberg, New York: Springer 1983

7. Metropolis, N., Stein, M.L., Stein, P.R.: On finite limit sets for transformations on the unit interval. J. Comb. Theory 15, 25-44 (1973)

8. Collet, P., Eckmann, J.-P.: Iterated maps on the interval as dynamical systems. Basel, Boston, Stuttgart: Birkhäuser 1980

9. Guckenheimer, J., Holmes, P.: Nonlinear oscillations, dynamical systems, and bifurcations of vector fields. Berlin, Heidelberg, New York: Springer 1983

Communicated by J.-P. Eckmann

Received December 23, 1985; in revised form July 14, 1986 
